Biologisches Institut der Universität Stuttgart

und Institut für Pharmazeutısche Biologie der Universität Bonn

\title{
Verhalten der Fettsäuren während der Entwicklung einiger Therophyten der Steppe von Kabul (Afghanistan)
}

\section{Fatty Acid Patterns During Development of Some Therophytes from the Plains near Kabul (Afghanistan)}

\author{
U. Kull und S.-W. Breckle
}

Mit 1 Abbildung

Eingegangen am 20. Januar 1975

\section{Summary}

The fatty acid composition of the saponifiable lipids of three therophytes Diarthron veszculosum, Scabiosa olvveri and Ziziphora tenutor from the plains near Kabul were investigated during development of the plants from the beginning of May until the end of June. In all three species, linolenic acid and hexadecenic acid contents decreased with increasing age. Zizıphora exhıbited greatest irregularities of fatty acid pattern. This species succumbs most rapidly with the onset of drought in June. Scabiosa had a high lipid content and the most balanced fatty acid pattern; this species is also more drought tolerant. This ecological advantage is discussed in relation to lipid metabolism. Diarthron was intermediate between the two species in these characteristics.

Key words: Fatty actds, Liptd metabolism, Therophytes.

\section{Einleitung}

Über das Verhalten der Fettsäuren der verseifbaren Lipide während des ganzen Entwicklungsganges von Therophyten sind uns keine Untersuchungen bekannt geworden, obwohl bei solchen Arten die Veränderungen im Stoffwechselgeschehen in kurzer Zeit ablaufen. Wir haben die Fettsäurezusammensetzung der Lipidfraktion aus den drei Arten Diarthron vesiculosum (Fisch. et C. A. MeY.) C. A. MeY., Scabiosa olıvieri Coult. und Zizıphora tenuoi L. während deren Entwicklung von Anfang Mai bis Ende Juni analysiert. Das bereits bezüglich der morphologischen Parameter und der osmotischen Verhältnisse (BRECKLE, 1971) und hinsichtlich der Mineralstoffe und verschiedener organischer Inhaltsstoffe (BRECKLE u. KulL, 1973) untersuchte Material der drei Therophytenarten aus der Steppe von Kabul bot sich zu derartigen Messungen an, zumal sich die Rohlipidfraktion bei den drei Arten unterschied- 
lich verhielt. So wurden bei Scabiosa Reservelipide vermutet (BreckLe u. Kull, 1973).

\section{Material und Methode}

Zu den Pflanzen und deren jewelligem Entwidklungszustand vgl. Breck1E (1971).

Die Extraktion der Lipide, die Aufarbeitung für dic Gaschromatographic und die qualitative und quantitative Bestimmung der Fettsauren erfolgte nach den bei KULL und JerEmias (1972) beschriebenen Verfahren.

\section{Ergebnisse}

In allen drei Arten konnten die meisten der enthaltenen Fettsäuren identifiziert werden. Die nicht oder nicht sicher identifizierten Peaks machen bei Zlziphora stets weniger als $9 \%$ der Gesamtfettsäuren aus, bei den beiden anderen Arten unter $5 \%$. Fettsäuren mit weniger als $14 \mathrm{C}$-Atomen haben stets nur geringe Mengenanteile und wurden in den Tabellen zusammengefaßt. Unter ihnen konnten stets Caprinsäure $(10: 0)$ und Laurinsäure $(12: 0)$ sowie mindestens eine ungesättigte Säure nachgewiesen werden. Bei älteren Diarthron-Pflanzen erscheinen in geringer Menge zwei Fettsäuren, deren Methylester sehr hohe Retentionszeiten (erheblich höher als Linolensäure) aufweisen. Sie sind vermutlich nur in den Samen enthalten und wurden nicht identifiziert.

Die Ergebnisse der quantitativen Bestimmungen sind in den Tabellen 1 bis 3 dargestellt. Die gemittelten Mengenveränderungen der Hauptfettsäuren zeigt die Abbildung.

Tab. 1: Fettsaurezusammensetzung der verseifbaren Lipide von Diarthron vesıculosum (in $\%$ der Gesamtfettsäuren).

\begin{tabular}{lrrrrrr}
\hline & \multicolumn{7}{c}{ Zeit } \\
Fettsäure & 8.5. & 20.5. & 1.6. & 10.6. & 16.6. & 28.6. \\
\hline$<14: 0$ & 6,9 & 4,4 & 1,9 & 2,4 & 1,9 & 1,9 \\
$14: 0$ & 3,1 & 2,8 & 0,4 & 0,3 & 0,2 & 0,1 \\
$14: 1$ & 1,6 & 2,9 & 1,2 & 1,4 & 0,7 & 1,1 \\
$16: 0$ & 33,6 & 44,9 & 33,0 & 23,6 & 14,1 & 17,5 \\
$16: 1$ & 3,1 & 2,9 & 1,5 & 0,8 & 0,4 & 0,2 \\
$18: 0$ & 2,8 & 5,6 & 6,4 & 4,5 & 2,8 & 3,8 \\
$18: 1$ & 7,1 & 9,3 & 18,2 & 17,7 & 18,6 & 21,5 \\
$18: 2$ & 9,6 & 7,5 & 17,7 & 32,0 & 49,4 & 43,9 \\
$18: 3$ & 31,7 & 17,6 & 16,9 & 13,1 & 8,1 & 5,8 \\
nicht identifiziert & 0,5 & 2,1 & 2,8 & 4,2 & 3,8 & 4,2 \\
molarer Quotient & & & & & & \\
$18: 2+18: 3$ & 1,13 & 0,51 & 0,96 & 1,75 & 3,73 & 2,60
\end{tabular}


Tab. 2: Fettsäurezusammensetzung der verseifbaren Lipide von Scabıosa olıvierl (in $\%$ der Gesamtfettsäuren).

\begin{tabular}{lrrrrrr}
\hline Fettsaure & 8.5. & 20.5. & $\begin{array}{c}\text { Zeit } \\
\text { 1.6. }\end{array}$ & 10.6. & 16.6. & 28.6. \\
\hline$<14: 0$ & 5,4 & 2,9 & 2,8 & 2,5 & 2,8 & 2,6 \\
$14: 0$ & 0,6 & 0,5 & 0,4 & 0,3 & 0,7 & 0,3 \\
$14: 1$ & 1,9 & 1,9 & 2,9 & 4,3 & 3,8 & 4,9 \\
$16: 0$ & 37,2 & 36,0 & 33,6 & 35,2 & 28,9 & 33,0 \\
$16: 1$ & 2,0 & 1,3 & 1,3 & 1,1 & 0,5 & 0,4 \\
$18: 0$ & 5,7 & 5,8 & 5,4 & 4,7 & 4,4 & 6,3 \\
$18: 1$ & 7,1 & 8,2 & 10,2 & 11,7 & 15,2 & 15,6 \\
$18: 2$ & 9,8 & 10,8 & 17,4 & 20,6 & 31,4 & 27,8 \\
$18: 3$ & 27,8 & 30,2 & 24,0 & 17,7 & 10,4 & 6,3 \\
nicht identifiziert & 2,5 & 2,4 & 2,0 & 1,9 & 1,9 & 2,8 \\
molarer Quotient & & & & & & \\
$18: 2+18: 3$ & 0,93 & 1,05 & 1,13 & 1,00 & 1,33 & 0,94 \\
\cline { 1 - 1 } $16: 0$ & & & & & &
\end{tabular}

Tab. 3: Fettsäurezusammensetzung der verseifbaren Lipide von Zıziphora tenuior (in \% der Gesamtfettsäuren).

\begin{tabular}{lrrrrrr}
\hline & \multicolumn{7}{c}{ Zeit } \\
Fettsaure & 8.5. & 20.5. & 1.6. & 10.6. & 16.6. & 28.6. \\
\hline$<14: 0$ & 1,7 & 2,3 & 3,2 & 2,3 & 2,6 & 2,4 \\
$14: 0$ & 0,4 & 0,8 & 0,4 & 0,3 & 0,4 & 0,3 \\
$14: 1$ & 1,3 & 3,1 & 2,4 & 1,4 & 1,3 & 1,8 \\
$16: 0$ & 32,4 & 44,4 & 33,7 & 30,3 & 29,4 & 21,1 \\
$16: 1$ & 2,5 & 2,2 & 0,5 & 0,1 & 0,2 & 0,1 \\
$18: 0$ & 5,4 & 6,6 & 7,4 & 7,1 & 7,9 & 6,3 \\
$18: 1$ & 8,3 & 9,1 & 20,1 & 20,2 & 30,2 & 28,7 \\
$18: 2$ & 13,0 & 12,0 & 10,8 & 9,9 & 4,2 & 9,6 \\
$18: 3$ & 32,3 & 16,1 & 16,4 & 21,6 & 15,4 & 22,8 \\
nicht identifiziert & 2,7 & 3,4 & 5,1 & 6,8 & 8,4 & 6,9 \\
molarer Quotient & & & & & & \\
$18: 2-18: 3$ & 1,28 & 0,58 & 0,74 & 0,96 & 0,61 & 1,41 \\
\cline { 1 - 3 } $16: 0$ & & & & & &
\end{tabular}

Scabıosa hat einen wesentlich höheren Lipidgehalt als die beiden anderen Arten. Jedoch treten keincrlei besondere Fettsäuren auf und auch dic quantitativen Veränderungen im Lauf der Untersuchungsperiode fügen sich in das Bıld der beiden anderen Arten ein. Bei Ztziphora werden ab Anfang Juni Unregelmäßigkeiten erkennbar, die bei den Ernten vom 16. 6. und 28. 6. sehr ausgeprägt sind. Sehr wahrscheinlich hängt dies mit dem früheren Absterben dieser Pflanzen zusammen. 


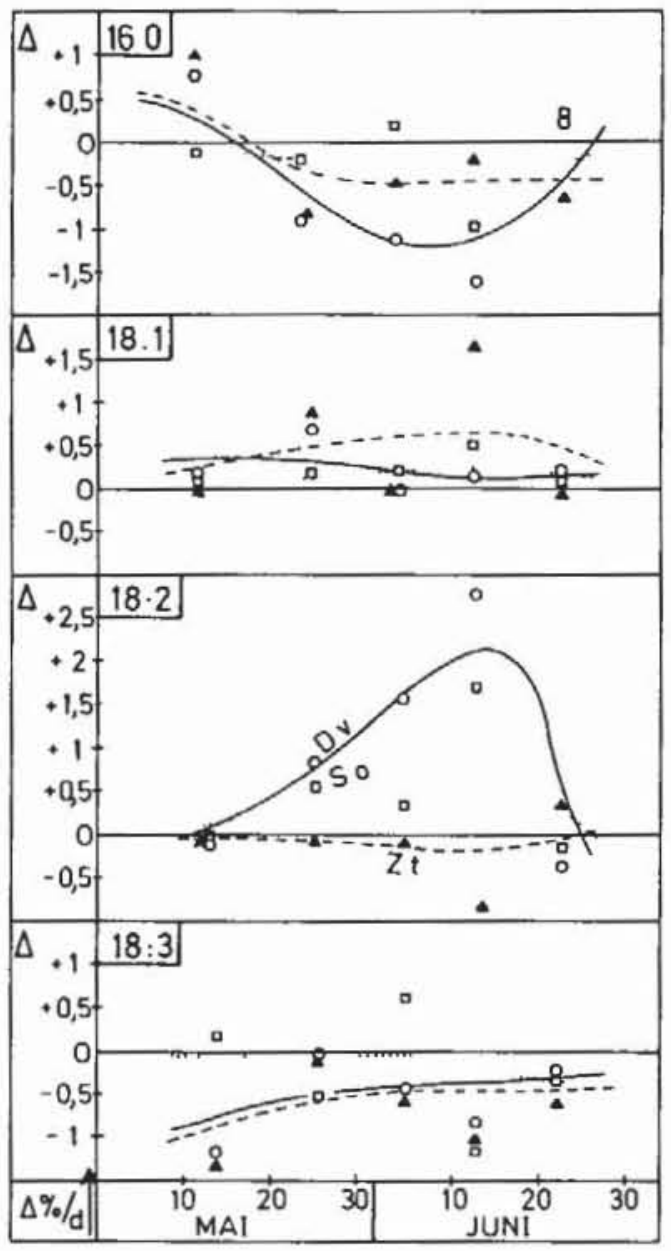

Abb. 1: Die Anderungen im Fettsäuregehalt der wichtıgsten Fettsauren (16:0 Palmitinsäure; 18:1 Olsäure; 18:2 Linolsäure; 18:3 Linolensäure) im Laufe der Entwidklung der drei untersuchten Therophyten (D. v.: Diarthron vesıculosum - Kreise, durchgezogene Linie; S. o.: Scabiosa olıvieri - Vierecke, punktierte Linı; Z. t.: Zızıphora tenuior - Dreicke, gestrichelte Linie). Als Ordinatenmaßstab angegeben ist dic tägliche Änderung des Prozentsatzes bezogen auf die Gesamtfettsäuren $(=100 \%$ ).

Linolensäure (18:3) nimmt bei allen drei Arten mit zunehmendem Pflanzenalter prozentual ab. Bei Scabiosa wird dieser Effekt erst im Verlauf des Juni deutlich, während er bei Diarthion und Ziziphora von Anfang an ausgeprägt ist. Das quantitativ geringere Ausmaß der Abnahme bei Ziziphora beruht darauf, daß bei dieser Art die Linolensäure in den Samen gespeichert wird, bei den beiden anderen hingegen nicht (vgl. Tabelle 6). Palmitinsäure (16:0) zeigt bei Diarthron und Ziziphora zu Beginn der Untersuchungsperiode eine prozentuale Mengenzunahme, danach kommt es zur Verringerung der Gehalte. Die Anteile von Linolsäure $(18: 2)$ und in geringerem Maß von OOlsäure (18:1) nehmen im Verlauf der Entwicklung bei Diarthron und Scabtosa zu. Bei Diarthron stellen diese beiden Komponenten in den letzten Entwicklungsstadien etwa $\% / 3$ der ganzen Fettsäurefraktion, in den jüngsten Stadien hingegen weniger als $1 / 5$. Bei Scabıosa nehmen sie von rund $1 / 5$ bis auf nahezu die Hälfte der 
Gesamtfettsäuren zu. Bei Zızıphora nimmt nur der Anteil der Olsäure zu, während der Prozentgehalt von Linolsäure sich langsam verringert.

Der Quotient Mol Linolsäure + Mol Linolensäure/Mol Palmitinsäure, der das Verhältnis des Hauptanteils mehrfach ungesättigter Fettsäuren zur Menge der wichtigsten gesättigten Fettsäure angibt, nimmt bei Dlarthron nach anfänglicher Abnahme stark zu. Bei Ztziphora zeigt er sehr unregelmäßige Schwankungen; bei Scabiosa steigt er zwar an, aber viel weniger ausgep:ägt als bei Diarthron.

Von okologischer Bedeutung ist das Gewicht der Samen und die Anzahl der Samen, die im Laufe einer Vegetationsperiode produziert werden. Allerdings spielen hierbei zahlreiche Faktoren eine Rolle, so daß im einzelnen ein Wettbewerbsvorteil fur die eine oder andere Art nicht ohne weiteres abgeleitet werden kann. In Tabelle 4 sind die Gewichte der Samen bəw. Früchte (Achänen, Nüßchen) wiedergegeben. Während sie bei Ziziphora schr einheitlich sind, schwanken sie bei Scabıosa stärker. In der Tabelle 5 sind die prozentualen Anteile der Samen bzw. Früchte an der oberirdischen Biomasse eines Individuums wiedergegeben. Im Lauf des Juni nimmt der Anteil durch "Aussaat” ab. Uber die Keimfähigkeit konnten wir keine Anhaltspunkte gewinnen; Samen aller drei Arten keımten auch bei sehr verschiedener Behandlung nicht.

Tab. 4: Gewicht einer Diaspore (Same bzw. Frucht) in mg. $\bar{m}$ : Mittelwert; s: Streuung (Standardabweichung, n: 5-12).

\begin{tabular}{lcccccr}
\hline Art & \multicolumn{7}{c}{ Zeit } & & & \\
& 1.6. & 10.6. & 16.6. & 28.6. & $\overline{\mathrm{m}}$ & $\mathrm{s}$ \\
\hline Diarthron v. & 0,35 & 0,52 & 0,59 & 0,41 & 0,47 & 0,16 \\
Scabiosa o. & 1,04 & 1,15 & 1,22 & 1,03 & 1,11 & 0,31 \\
Ziziphora t. & 0,28 & 0,27 & 0,33 & 0,28 & 0,29 & 0,06
\end{tabular}

Tab. 5: Prozentualer Anteil der Diasporen (Samen bzw. Früchte) an der oberirdischen Biomasse (Trockengewicht).

\begin{tabular}{lllrrrrr}
\hline Art & 8.5 & 20.5 & 1.6. & 10.6. & 16.6. & 28.6. \\
\cline { 1 - 4 } Diarthron v. & 0 & 0 & 9 & 28 & 25 & 16 \\
Scabiosa o. & 0 & 0 & 20 & 24 & 29 & 21 \\
Ziziphora t. & 0 & 0 & 3 & 13 & 14 & 8
\end{tabular}

Da der Anteil der bei allen drei Arten fettreichen Samen an der oberirdischen Biomasse bei Diarthron und Scabiosa auf mehr als $1 / 4$ ansteigt, schien es wichtig, die Fettsäuremuster der Samenlipide ebenfalls zu untersuchen. Die Befunde sind in Tabelle 6 dargestellt. Bei Zızıphora herrscht Linolensäure mengenmäßig vor. Bei Diarthron und Scabıosa steht hingegen Linolsäure an erster Stelle, gefolgt von Olsäure. Sicher ist die Zunahme der Linolsäure bei diesen beiden Arten mit der Alterung der Pflanzen, auf die Samenbildung zurückzuführen; für Olsäure wird dies zum Teil auch zutreffen. 
Tab. 6: Fettsaurezusammensetzung der verseifbaren Lipide der Diasporen (Samen bzw. Früchte) in \% der Gesamtfettsäuren.

\begin{tabular}{|c|c|c|c|}
\hline \multirow[t]{2}{*}{ Fettsäure } & \multicolumn{3}{|c|}{ Art } \\
\hline & Diarthron $v$. & Scabiosa o. & Zlziphorat. \\
\hline$<14: 0$ & 0,4 & 0,6 & 0,3 \\
\hline $14: 0$ & 0,3 & 2,3 & 0,2 \\
\hline $16: 0$ & 7,7 & 11,1 & 6,3 \\
\hline $18: 0$ & 2,5 & 3,4 & 2,6 \\
\hline $18: 1$ & 15,9 & 13,9 & 9,0 \\
\hline $18: 2$ & 67,4 & 66,1 & 16,5 \\
\hline $18: 3$ & 2,2 & 1,7 & 64,5 \\
\hline sonstige & 3,6 & 0,9 & 0,6 \\
\hline
\end{tabular}

\section{Diskussion}

Die Ergebnisse zeigen, daß sich das Fettsäuremuster im Verlauf der Entwicklung der Pflanzen quantitativ erheblich verändert. Auffällig ist besonders der mit zunehmendem Pflanzenalter abnehmende Anteil von Linolensäure bei allen Arten. Gleichartig verhält sich Hexadecensäure (16:1); allerdings sind ihre Absolutgehalte viel geringer und somit die Meßfehler höher. Wenn man dies berücksichtigt, so ist die Korrelation zwischen der Alterung der Pflanzen und der Abnahme des prozentualen Gehalts an Hexadecensäure überraschend gut. Die Abnahme erfolgt bei Ziziphora am raschesten, bei Scabiosa am langsamsten. Dies entspricht völlig den morphologischen Befunden von BRECKLE (1971). Möglicherweise deuten diese Mengenveränderungen auf die Abnahme der Funktionsfähigkeit der Chloroplasten hin, die der zunehmenden Dürreschädigung parallel verläuft.

Unregelmäßigkeiten in den Gehaltsveränderungen, die durch das Absterben von Pflanzenteilen bedingt sein dürften, treten, wie schon erwähnt, zuerst bei Ztziphora auf und sind bei Diartbron Ende Juni ebenfalls zu erkennen. Bei Scabiosa lassen sie sich bis zum Ende der Probennahme nicht feststellen. Dies ist - neben dem Verhalten von Linolen- und Hexadecensäure - ein weiterer Hinweis darauf, daß Scabiosa einen besonders ausgeglichenen Fettsäurehaushalt aufweist. Der gegenüber den beiden anderen Arten wesentlich höhere Lipidgehalt (BRECKLE und KULL, 1973) hat sicher physiologische und ökologische Bedeutung. So könnte man etwa die auch noch Ende Juni feststellbare Neubildung von Blütenständen und damit weiterer Verbreitungseinheiten in einer Zeit, in der die Pflanze kaum noch Wasser aus dem Boden aufnehmen kann und die Stoffbilanz negativ ist, damit in Verbindung bringen. Interne Umlagerungen gespeicherter Substanzen werden hier vermutlich bis zuletzt zur Bildung von Diasporen ausgenutzt. Sicher steht der ausgeglichene Lipidhaushalt mit der relativ langen Lebensfähigkeit der Scabiosa-Individuen im Zusammenhang. LARCHER et al. (1973) haben darauf hingewiesen, daß eine Speicherung von Reservestoffen in der energiereichen Form von Lipiden sehr günstig sein kann, da diese Substanzen in ihrer 
Ablagerungsform weniger temperaturabhängig sind als Kohlenhydrate. In produktionsungünstigen Zeiten kann ein hoher Zuckerspiegel sogar nachteilig sein wegen einer möglicherweise intensiveren Atmung (vgl. LARCHER et al., 1973).

Der molare Quotient 18:2+18:3/16:0 steigt bei Scabiosa viel weniger stark an als bei Diarthron, was vielleicht auf eine geringere Stress-Empfindlichkeit des Lipidstoffwechsels hindeutet. Die geringeren Werte des Quotienten und sein unregelmäßiges Verhalten bei Ziziphora sind dadurch verursacht, daß bei dieser Art auch die Linolsäure mit zunehmendem Pflanzenalter abnimmt.

Ein eindeutiger Einfluß der Temperatur auf das Fettsäuremuster ist nicht festzustellen. Allerdings herrschte während der Zeit der Probennahmen nic ausgesprochene Kälte (vgl. BRECKLE, 1971), die auf den Fettsäurehaushalt deutliche Auswirkungen hätte haben können (Kull u. Jeremias, 1972). Die Beeinflussung durch hohe Temperaturen ist zumindest bei Atriplex gering (Pearcy u. Strouse, 1974). Da außerdem im Juni Wassermangel, hohe Temperatur bei großer Tagesamplitude und natürliche Pflanzenalterung (Frucht- und Samenbildung) unter Feldbedingungen gleichzeitig wirksam sind, lassen sich genauere Aussagen hierzu nicht machen.

Für die sorgfältige Durchführung der gaschromatographischen Analysen danken wir Fräulein Barbara Kuhn, Stuttgart; fur sonstige technische Assistenz Fraulein Irmingard Meier, Bonn.

\section{Literatur}

Brecki.e, S.-W.: Ist Diarthron vesiculosum (Thymelaeaceae) ein ökologisches Ratsel? I. Allgemeines, Morphologie und osmotische Verhältnisse. Botan. Jb. 90, 550-561 (1971).

Breckle, S.-W., und U. Kul.L: Ist Diarthron vestculosum (Thymelaeaceae) ein ökologisches Rätsel? II. Die Wirkung der Dürre auf Mineralstoffverhältnisse und Kohlenhydrathaushait. Botan. Jb. 93, 539-561 (1973).

Kull, U., und K. Jeremias: Die Fettsäurezusammensetzung der verseifbaren Lipide aus Rinden von Populus balsamifera im Jahresgang. Z. Pflanzenphysiol. 68, 55-62 (1972).

Larcher, W., L. Schmid r und A. Tschager: Starke Fettspeicherung und hoher Kaloriengehalt bei Lotseleura procumbens (L.) Desv. Oecol. Plant. 8, 377-383 (1973).

Pearcy, R. W., and B. Strouse: Growth temperature effects on leaf lipids in coastal and desert races of Atriplex lentiformis. Plant Physiol. 53, Ann. Suppl. 34 (1974).

Dr. U. Kulı, Biologisches Institut der Univ. Stuttgart, D-7000 Stuttgart 60, Ulmer Str. 227.

Dr. S.-W. Breckle, Institut für Pharmazeutische Biologie, D-5300 Bonn, Nußallee 6. 